Interactive comment on "Stratospheric aerosol layer perturbation caused by the $\mathbf{2 0 1 9}$ Raikoke and Ulawun eruptions and climate impact" by Corinna

\title{
Kloss et al.
}

\section{Corinna Kloss et al.}

corinna.kloss@cnrs-orleans.fr

Received and published: 4 November 2020

Please see attached file.

Please also note the supplement to this comment:

https://acp.copernicus.org/preprints/acp-2020-701/acp-2020-701-AC2-supplement.pdf 